Projets

de paysage

\section{Projets de paysage}

Revue scientifique sur la conception et l'aménagement de l'espace

$17 \mid 2017$

Paysage(s) et agriculture(s)

\title{
Le paysage agricole comme médiation dans la communication du parc naturel régional du Pilat
}

Diversité, double contrainte et patrimonialisation

The Agricultural Landscape as a Mediator in the Communication of the Pilat Regional Nature Park - Diversity, Double Constraint and Heritagization

\section{Émilie Kohlmann}

\section{OpenEdition \\ Journals}

Édition électronique

URL : http://journals.openedition.org/paysage/4642

DOI : 10.4000/paysage.4642

ISSN : 1969-6124

\section{Éditeur :}

École nationale supérieure du paysage de Versailles-Marseille, Institut national des sciences appliquées Centre Val de Loire - École de la nature et du paysage, École nationale supérieure d'architecture et de paysage de Bordeaux, École nationale supérieure d'architecture et de paysage de Lille, Agrocampus Angers

Référence électronique

Émilie Kohlmann, « Le paysage agricole comme médiation dans la communication du parc naturel régional du Pilat », Projets de paysage [En ligne], 17 | 2017, mis en ligne le, consulté le 03 avril 2020. URL : http://journals.openedition.org/paysage/4642 ; DOI : https://doi.org/10.4000/paysage.4642

Ce document a été généré automatiquement le 3 avril 2020.

Projets de paysage 


\title{
Le paysage agricole comme médiation dans la communication $\mathrm{du}$ parc naturel régional du Pilat
}

\author{
Diversité, double contrainte et patrimonialisation
}

The Agricultural Landscape as a Mediator in the Communication of the Pilat Regional Nature Park - Diversity, Double Constraint and Heritagization

\section{Émilie Kohlmann}

«Le paysage est la résultante des interrelations
entre les activités humaines, les facteurs naturels
et des représentations individuelles et collectives.
L'activité agricole fait partie de celles qui jouent
un rôle majeur en ce domaine. » (Source : Dossier
documentaire « Prairies fleuries : des ressources
agricoles à valeur écologique ", Parc naturel
régional du Pilat, 2014.)

1 Cette première définition, extraite d'un document de communication du Parc naturel régional (PNR) du Pilat servira de point de départ à cet article : le paysage tel qu'il est défini par les acteurs du territoire. Il y apparaît comme une interrelation complexe entre aspects humains et environnementaux, interrelation qui comporte une partie incompressible de réel mais également de représentations. Cette conception correspond à celle donnée par la Convention européenne du paysage où il est considéré comme « une partie de territoire telle que perçue par les populations, dont le caractère résulte de l'action de facteurs naturels et/ou humains et de leurs interrelations ${ }^{1} »$. Le paysage sera pris comme l'interrelation du milieu physique et du milieu social, irréductible à l'un ou à l'autre de ces deux aspects qui, en permanence, s'influencent mutuellement (Berque, 2000).

2 Le PNR du Pilat souligne la part importante qu'il souhaite accorder à l'activité agricole en tant que productrice de paysage de nature ordinaire. Ce dernier point n'est pas 
anodin : il rappelle la différence entre parcs régionaux et nationaux dans leur rapport au caractère remarquable de leurs paysages et de leurs écosystèmes. Les parcs nationaux valorisent et communiquent traditionnellement plus autour de paysages remarquables que les parcs régionaux qui mettent en scène une nature ordinaire (Larrère, Lizet et Berlan-Darqué, 2009).

3 Les concepts et méthodes issus des sciences de l'information et de la communication permettent d'observer comment le parc a élaboré une stratégie de communication dans laquelle les pratiques agricoles peuvent être valorisées à travers le paysage. Ce dernier devient un espace de médiation qui permet de porter ensemble différents propos, parfois difficilement conciliables. Le thème de la diversité devient alors central pour faire tenir ensemble acteurs humains et non humains dans la communication d'une institution en charge de la protection de son patrimoine naturel et culturel.

Nous commencerons par présenter plus en détail le contexte de recherche qui s'appuie sur une thèse sur la communication environnementale d'un PNR en région RhôneAlpes, le Pilat. Puis, nous reviendrons sur les trois entrées théoriques qui ont été mobilisées plus précisément pour l'analyse de la communication autour des paysages agricoles sur le territoire : la diversité comme outil de valorisation de l'agriculture, la mise en avant de certaines pratiques considérées comme vertueuses sur le plan environnemental et le recours au patrimoine. Enfin, j'illustrerai ces trois points par les trois figures principales mobilisées par le Parc dans sa communication: le tracteur comme symbole duel des pratiques agricoles, les prairies fleuries et la double contrainte communicationnelle ${ }^{2}$, le troupeau et la lande comme processus de patrimonialisation paysagère et agricole.

\section{Le PNR : un acteur historique important sur le territoire du Pilat}

\section{Diversité vs caractère remarquable}

5 En 1974, la région Rhône-Alpes acte par décret la naissance du Parc naturel régional du Pilat. Situé à 90 \% sur le département de la Loire et à $10 \%$ sur celui du Rhône, ce Parc s'étend sur une superficie de $700 \mathrm{~km}^{2}$. Il comprend 47 communes rurales pour un total d'environ 57000 habitants. Son caractère périurbain est marqué par la proximité de métropoles comme Lyon ou Saint-Étienne ou de villes de moyenne importance comme Annonay en Ardèche. C'est d'ailleurs une des raisons de son existence puisque son dossier de création a été porté par deux notables stéphanois pour répondre à une crainte liée à l'urbanisation galopante et aux atteintes, notamment paysagères, portées à un espace de nature récréatif ${ }^{3}$.

6 D'un point de vue géomorphologique, il s'est construit autour d'un massif de moyenne montagne dans lequel trois climats se rencontrent (influences méditerranéennes, océaniques et continentales). Six entités paysagères sont identifiées par le PNR dans ses représentations du territoire : le versant Gier, le piémont rhodanien, les Crêts, la vallée de la Déôme, le Haut-Pilat et la côtière rhodanienne ${ }^{4}$. Cette diversité paysagère, climatique, écologique, etc., fait partie des éléments mis en avant par la collectivité elle-même. Pourtant, dans les entretiens menés auprès des différents chargés de mission du Parc ${ }^{5}$ (Kohlmann, 2016), nombreux sont ceux qui regrettent la pauvreté du territoire en matière d'éléments paysagers ou naturels emblématiques. Leurs propos 
ont tendance à souligner la difficulté qu'il y a à communiquer sur du quotidien, de l'ordinaire, au regard des critères esthétiques ou touristiques habituels qui s'appuient sur du remarquable.

« Nous, on n'a pas de grande réserve naturelle, on n'a pas un animal emblématique, super-protégé que tout le monde nous envie. Voilà, donc dans le réseau des Parcs et des espaces naturels protégés, le Pilat n'était pas reconnu, n'avait pas une réputation d'être un milieu naturel exceptionnel ${ }^{6}$. " (Chargée de communication du PNR, avril 2011.)

7 Cette tension entre aspiration au remarquable et constat de son absence permet de mieux appréhender la stratégie de communication mise en place. Elle substitue ainsi la valorisation de l'ordinaire dans sa diversité à la représentation du remarquable dans son unicité comme nous le verrons par la suite à travers différents exemples.

\section{Une des missions du PNR : la valorisation de l'agriculture}

Les PNR ont des missions définies dans une charte renouvelée tous les douze ans et signée par les différents acteurs du territoire : État, région, communes, etc. Entre 2010 et 2015, période durant laquelle s'est déroulée l'étude, le Pilat a procédé à la réécriture de sa charte. La nouvelle est entrée en vigueur en 2013. Elle s'intitule : «Charte objectif 2025 : rapport et annexes ${ }^{7} »$. Elle comporte parmi ses différents axes de travail culturels, sociaux ou environnementaux un axe 3 qui ambitionne de « revaloriser le métier d'agriculteur ».

«L'agriculture pilatoise, forte de ses 90 millions d'euros de produit annuel brut, renforce l'économie locale. Ce secteur est pourtant menacé : le métier d'agriculteur n'attire que peu de jeunes et certaines entreprises agricoles de taille modeste peinent à survivre. Conscient que l'agriculture de proximité est un atout indéniable pour le cadre de vie et l'environnement du massif, le Parc soutient les agriculteurs. » (Résumé synthétique « La charte objectif 2025, septembre 2011.)

Des actions concrètes sont mises en place par le PNR pour guider les agriculteurs dans leur pratique: formations, aide au montage de dossiers de financement pour les Mesures agro-environnementales (MAE), mise en place d'un observatoire agricole de la biodiversité, accompagnement des agriculteurs dans leurs démarches de certification environnementale, etc. La communication du Parc participe d'une stratégie plus globale de valorisation et d'accompagnement de l'agriculture.

10 En 2010, le PNR abritait 953 exploitations agricoles, dont les trois quarts sont des exploitations individuelles, les autres étant sous formes sociétaires comme les Groupements agricoles d'exploitation en commun (GAEC). La moitié des exploitations du Parc sont de petites tailles avec un chiffre d'affaires potentiel inférieur à $25000 €$. $81 \%$ exploitent moins de 50 hectares et seulement $1 \%$ plus de 100 . La moyenne de la surface agricole utile (SAU) est de 26 hectares.

11 L'agriculture est plutôt de type extensif, notamment à cause des caractéristiques géomorphologiques évoquées plus haut: peu de grandes plaines, de fortes pentes, des parcelles parfois morcelées, voire inaccessibles à la mécanisation.

«Les structures sur le Pilat, on a quand même des gens qui font une agriculture à peu près extensive, qui ont des tas de problèmes, des petits revenus... et pour nous le fait qu'ils restent en place de toute façon, c'est un élément de biodiversité à préserver quoi qu'il arrive. Moi j'ai moins de problèmes et de difficultés avec l'agriculture dans le Pilat qu'ailleurs 8 . » (Membre de la Fédération Rhône-Alpes de protection de la nature Loire [Frapna], octobre 2013.) 
12 Enfin, si l'élevage est très présent sur le territoire, certains secteurs se sont néanmoins spécialisés : lait de vache sur Saint-Genest-Malifaux et Bourg-Argental ou encore vins et fruits autour de Pélussin et de Condrieu. Le soutien à l'agriculture semble s'inscrire dans un contexte favorable à la mise en avant de la diversité des pratiques, des paysages et des écosystèmes.

\section{Une communication institutionnelle qui associe fréquemment paysage, agriculture et environnement}

13 Cet article s'appuie sur un ensemble varié de matériaux (observations participantes, analyse de corpus qui croise productions matérielles et numériques du Parc avec le suivi de ses sites Internet et le recueil de ses publications et autres goodies, entretiens semi-directifs). À travers leur analyse, il apparaît que les représentations de l'agriculture et des paysages agricoles dans la communication du Parc alimentent un discours plus large sur la richesse écologique du territoire. Nous retrouvons ainsi imbriqués sur un même terrain: pratiques agricoles, maintien de patrimoines paysagers, richesse en biodiversité et identité d'une collectivité territoriale.

À cette occasion, les questions environnementales ont été abordées à travers le thème de la biodiversité, très actif à l'époque du travail de recherche ${ }^{9}$. Nous avons pu observer le rapprochement fréquent des thèmes de biodiversité et d'agriculture : structurellement d'abord à travers le fonctionnement organisationnel du PNR (réunions croisées des acteurs, organigramme, etc.), mais aussi à travers la communication événementielle (lancement de la première "Journée de l'observatoire de la biodiversité » qui mélange naturalistes et agriculteurs par exemple), et enfin à travers les documents de communication eux-mêmes (dossiers documentaires, communication sur les "prairies fleuries ", journal institutionnel du parc, L'Écho des chirats). Ce sont ces derniers qui seront plus particulièrement mobilisés dans cet article. On se penchera de manière plus précise sur ce lien entre paysage et agriculture dans la communication du PNR du Pilat.

Il semble nécessaire d'introduire dans ce couple deux autres notions: celles de patrimoine et de diversité environnementale et culturelle. Nous reprendrons les conclusions déjà émises lors de la thèse, mais en faisant ici le choix d'entrer plus dans le détail d'une analyse sémiologique visuelle des paysages mobilisés dans les documents de communication.

Le corpus d'étude retenu s'appuie sur la collection des Dossiers documentaires du Parc, particulièrement probants dans leur usage des photographies et des infographies paysagères agricoles ${ }^{10}$. Nous $\mathrm{y}$ adjoindrons les dispositifs de communication mis en place autour de la thématique des "prairies fleuries", conçue initialement à destination des agriculteurs au niveau national par la Fédération des PNR. Elle a par la suite été réinvestie par le Parc du Pilat dans une communication plus grand public. Le lien avec la thématique initiale de la biodiversité se fera à partir du thème de la diversité de manière plus large. 


\section{Diversité, pratiques agricoles « vertueuses » et patrimoine rural}

\section{La diversité comme figure positive pour valoriser l'agriculture}

Si nous avons insisté sur la mission du PNR quant à la défense de la profession agricole inscrite dans sa charte, c'est parce qu'il semble important de prendre en compte l'évolution de cette profession depuis plusieurs décennies. Le modèle productiviste et monofonctionnel commence à être remis en question, notamment dans les territoires pour lesquels il a toujours été inadapté. C'est le cas du Pilat qui, géomorphologiquement, ne peut mettre en place une agriculture identique à celle présente dans la plaine stéphanoise : petites parcelles, inaccessibles, en forte pente, etc. Le modèle d'une agriculture postproductiviste et multifonctionnelle y est donc favorisé (Ruiz et Domon, 2014). L'agriculture continuerait donc de jouer un rôle traditionnel de production agroalimentaire, tout en se diversifiant et en s'insérant dans d'autres pans de l'économie locale: tourisme, consommation et protection de l'environnement, notamment à travers son impact paysager (ibid.). L'idée de diversité comme outil de valorisation des pratiques agricoles apparaît peu à peu dans ce postulat. Or, l'image précédente d'une agriculture intensive, de type monoculture, détériorant les paysages en les uniformisant est encore très présente dans les représentations de celle-ci.

Il semblerait que dans sa rhétorique, le PNR du Pilat contribue à mettre la diversité au centre de la valorisation de l'agriculture selon le schéma narratif suivant :

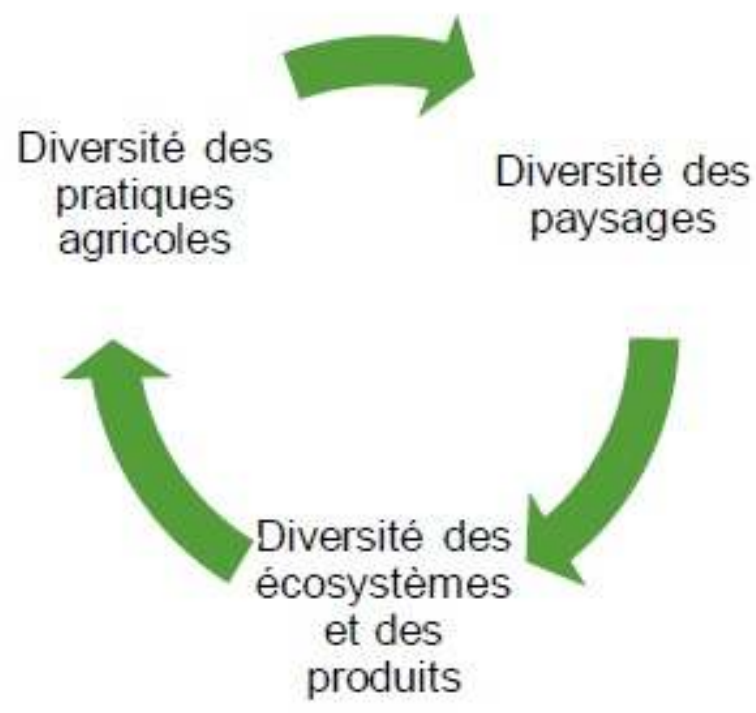

La thématique de la diversité est filée comme stratégie de communication et de revalorisation de l'agriculture. Elle est l'un des leviers employés par le Parc pour améliorer les représentations de la profession et de ses pratiques. Nous rejoindrons ici la conclusion de Raphaël Larrère, Bernadette Lizet et Martine Berlan-Darqué (2009) au sujet des parcs naturels nationaux. Selon les nouveaux paradigmes mobilisés dans la gestion de la nature, ce n'est plus l'activité humaine en elle-même qui est une menace sur celle-ci, mais sa standardisation. La diversité, notamment culturelle, devient ainsi une valeur positive, à favoriser et à mettre en avant. Dans un article de 2011, Florent 
Kohler critique ce lien causal implicitement marqué entre diversité culturelle et maintien de la biodiversité. Selon lui, le rapport entre les deux est plus complexe et moins linéaire qu'un simple rapport de cause à effet. Le débat entre pairs qui a suivi dans la revue Natures Sciences et Sociétés illustre la difficulté à remettre en question ce lien tout comme la valorisation de ces deux aspects.

\section{Des pratiques agricoles présentées comme vertueuses et favorables à l'environnement}

20 Protection de la nature et esthétisme paysager sont historiquement associés (Larrère, Lizet et Berlan-Darqué, 2009). À la fin du XIX siècle, c'est pour préserver de l'homme les paysages sauvages américains, la wilderness, que furent créés les premiers parcs nationaux ${ }^{11}$. En France, au début du $\mathrm{Xx}^{\mathrm{e}}$ siècle, les lois paysage furent les premières lois à permettre la protection de sites naturels et elles l'autorisèrent sur des critères esthétiques $^{12}$. Ces paysages protégés l'étaient initialement de l'homme et de ses activités. La prise en considération des paysages agricoles est plus récente et ne semble pas s'appuyer sur les mêmes mécanismes. En effet, à mille lieues des paysages pittoresques ou sublimes valorisés à travers la wilderness à la fin du XIX ${ }^{e}$ siècle, les paysages agricoles se rattachent à des paysages ordinaires (Ruiz et Domon, 2014). Loin de devoir en être rejetée, l'activité humaine est présentée comme étant à l'origine de ces paysages. Les interventions humaines, comme le pâturage, participent au maintien des écosystèmes en place (Lepart et Marty, 2006).

21 C'est dans les années 1990 que le thème de l'environnement est pris en considération dans le renouvellement des politiques agricoles au niveau national mais également européen avec des actions comme Natura 2000 ou les mesures agro-environnementales (Poux, 2006). En effet, l'agriculture participe à la gestion de la diversité du vivant (Bonneuil et Fenzi, 2011). Elle est impliquée dans les projets de gestion de la biodiversité, notamment à travers la sélection de pratiques jugées favorables au maintien de la diversité des écosystèmes. Lier biodiversité et rôle des savoirs traditionnels permet de revaloriser ces derniers (Roué, 2003 ou Kohler, 2011). Cette association donne aux paysages une valeur écosystémique (Lucas et Maisonhaute, dans Ruiz et Domon, 2014) et non plus simplement esthétique. Elle les inclut dans des systèmes écologiques plus globaux.

22 L'idée que les pratiques agricoles vertueuses au niveau environnemental sur le territoire sont la raison d'une biodiversité riche et préservée se retrouve ainsi dans les discours du PNR du Pilat. Nous proposons alors de faire l'hypothèse que le paysage y est mobilisé comme un espace de médiation. Il autorise l'articulation de deux éléments : pratiques agricoles et biodiversité riche, et de deux types d'acteurs: naturalistes et agriculteurs, qui se rejoignent à travers lui.

\section{Esthétisme d'un paysage rural patrimonialisé}

Enfin, les parcs naturels régionaux ont vocation à participer à la conservation du patrimoine territorial et les pratiques agricoles sont régulièrement reconstruites et réinscrites dans une tradition territoriale à travers la communication du PNR du Pilat. Le patrimoine est ici entendu comme un fait social, un processus au cours duquel des 
choix sont opérés: choix de l'objet, choix de ses représentations, de l'histoire construite autour de lui, etc.

«[...] peu de recherches considèrent le patrimoine comme un fait social qu'il conviendrait d'étudier en tant que tel. Un nombre encore plus restreint l'aborde comme un fait communicationnel, c'est-à-dire comme un processus dans lequel l'objet patrimonial est le support d'une relation entre celui qui le met en valeur et le visiteur (comme un support de médiatisation), tout en étant l'opérateur par lequel se construit un lien entre nous qui en avons l'usage et ceux qui l'ont produit (c'est-à-dire un opérateur de médiation).»(Davallon, 2006, p. 16.) ne peuvent être conciliées. (Guérin, 1995).

La médiatisation du paysage agricole comme patrimoine du territoire du PNR fait de celui-ci un opérateur de médiation. Il devient le moyen de faire cohabiter des attentes communicationnelles parfois antinomiques ou difficilement conciliables. Nous renvoyons ici à la notion de double bind en psychologie de la communication. La communication paradoxale (Watzlawick, Beavin et Jackson, 1972) met en avant les phénomènes de communication qui s'opposent les uns aux autres, dont les deux parties

Dans l'exemple du paysage agricole, nous pouvons nous interroger sur la manière de faire cohabiter les représentations d'un paysage rural supposé déterritorialisé et esthétisé par les non-agriculteurs (Poinsot, 2008) et les contraintes concrètes des acteurs dans le cadre de leur pratique professionnelle. Cette question sera plus particulièrement étudiée à travers le cas des prairies fleuries.

Pourtant, reconstruire une image patrimoniale des pratiques agricoles d'un territoire n'est pas sans bénéfices. Cela permet notamment de valoriser les productions locales comme non transférables (Cevasco et Poggi, 2000) car liées à un savoir-faire local et à un environnement typique. Quant aux paysages produits par une certaine agriculture, leur dimension esthétique en fait également un enjeu touristique et économique

27 Le paysage agricole, figure emblématique d'un certain imaginaire de la ruralité, peut alors devenir un enjeu pour les collectivités, notamment lorsqu'elles ont à construire et à maintenir une identité particulière: celle d'institutions patrimoniales à vocation environnementale comme c'est le cas pour les parcs naturels régionaux (Micoud, Laneyrie et Banville, 1977; Baron et Lajarge, 2015). Le Parc naturel des Causses du Quercy a ainsi fait de son paysage ordinaire, issu de l'agriculture, un projet et une perspective de développement durable du territoire (Ruiz et Domon, 2014)

Comme Jean Davallon (2006), nous considérons que la patrimonialisation est un processus réalisé par des acteurs qui choisissent les éléments retenus. Cet auteur accorde un statut performatif à la déclaration d'un objet comme patrimoine :

«La question de ce que pouvait signifier « être patrimoine » s'est posée dans toute sa radicalité avec l'élargissement des patrimoines, avec l'émergence du patrimoine vernaculaire, du patrimoine immatériel de la mémoire, du patrimoine naturel, etc. [...] C'est «nous » qui décidons que tels outils, telles usines, tels paysages, tels discours ou telles mémoires, doivent acquérir le statut de patrimoine. Le présupposé selon lequel le patrimoine est un donné, dont le statut préexisterait et se transmettrait simplement du passé vers le présent, n'est en ce cas absolument pas tenable. » (Davallon, 2006, p. 96.)

Choisir de mettre en place une communication où les pratiques agricoles sont présentées comme vertueuses en matière d'environnement lorsqu'elles sont plurifonctionnelles, peu mécanisées, extensives et attachées à des éléments de bâtis 
vernaculaires comme les murets de pierres sèches, les haies, etc., contribue à un mécanisme de patrimonialisation. Ce dernier construit une image positive du territoire qui, en outre, est défendable sur les plans économique et touristique. Cet aspect sera plus particulièrement observé à travers la figure du troupeau et de la lande en dernière partie.

\section{Valorisation de la diversité dans les paysages agricoles mobilisés dans la communication du PNR du Pilat}

\section{Le tracteur et les pratiques : valorisation de la diversité}

La figure du tracteur sera étudiée en premier lieu pour exprimer la mise en valeur de la thématique de la diversité dans la communication du PNR du Pilat. Dans les documents étudiés ici, les Dossiers documentaires, le tracteur apparaît régulièrement dans des photographies de paysage. Élément motorisé et visuellement représentatif, il symbolise la pratique agricole et est également repris à ce titre dans les infographies réalisées.

Figure 1. La figure du tracteur comme représentation d'une activité agricole intensive destructrice des " habitats naturels " et responsable de la " disparition des prairies naturelles "

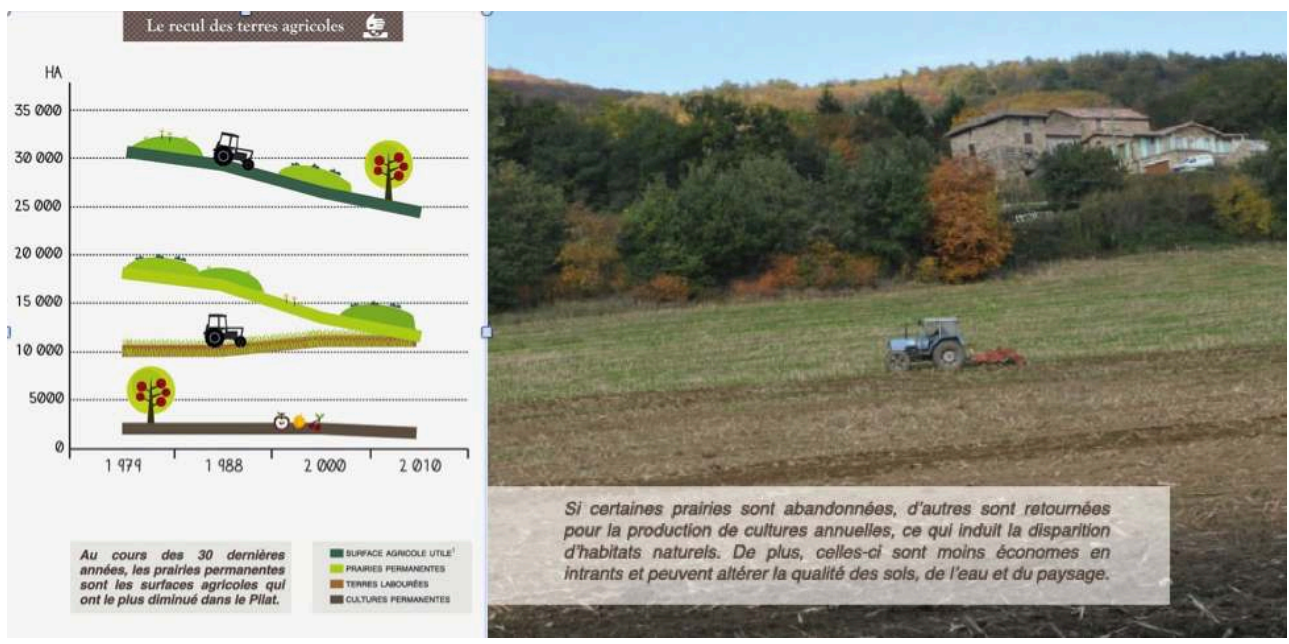

Dossier documentaire : "L'agriculture dans le Pilat ».

Source : images extraites de documents édités par le Parc naturel régional du Pilat (RGA 2000 et 2010/PNR Pilat - Observatoire de l'agriculture du Pilat).

31 À travers ce premier exemple, extrait d'une seule et même page du Dossier documentaire consacré à l'agriculture dans le Pilat, le tracteur est d'abord le symbole de la mécanisation et d'une agriculture intensive et peu respectueuse de l'environnement. Dans le texte qui légende la photographie ci-dessus, le vocabulaire ne laisse aucun doute : les prairies sont « abandonnées » ou "retournées », ce qui cause la "disparition d'habitats naturels ", elles sont alors "moins économes» et de qualité " altér[ée] ». Quant à l'image en elle-même, elle montre un champ nu ou presque, sans aucune diversité, avec en arrière-plan une forêt et des habitats. Nous verrons par la suite combien cela tranche avec les représentations paysagères considérées comme positives dans les documents de communication du Parc. 
Dans la figure 2, extraite du dossier sur les prairies fleuries, le tracteur est représenté deux fois dans les photographies, en action dans des paysages peu diversifiés. Il est associé à un texte qui évoque une «menace » sur certains types d'habitats favorables à l'environnement, dont l'agriculture intensive cause la « disparition ».

Figure 2. Par métonymie, le tracteur évoque l'ensemble des pratiques agricoles considérées comme négatives : mécanisation, labour, drainage des terres, etc.

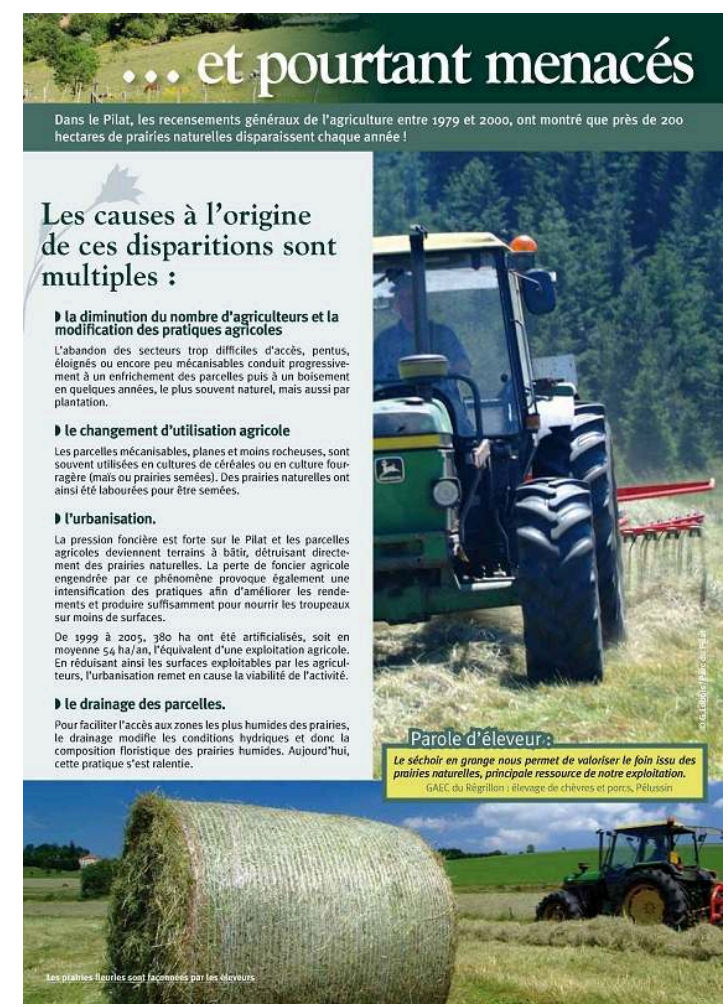

Source : image extraite de documents édités par le Parc naturel régional du Pilat.

Mais la figure du tracteur est plus complexe qu'il n'y paraît si on ne s'arrête pas à ces premiers exemples. En effet, il peut également être mobilisé pour représenter une autre agriculture, une agriculture aux pratiques vertueuses et respectueuses de l'environnement. Il est alors départi de ses attributs négatifs: monoculture, mécanisation, agriculture intensive, pour ne plus représenter que de manière neutre l'activité agricole.

Dans le dossier consacré aux prairies fleuries, dès la couverture, le tracteur est mis en liaison avec un paysage de prairie couvert de fleurs, coloré et connoté positivement. La relation entre les figures de l'abeille et de la fleur, qui représentent alors la diversité biologique, et le tracteur se met en place à travers le paysage comme médiation. C'est une autre forme de pratique agricole, extensive et respectueuse de l'environnement, qui est mise en scène : celle qui permet la création et le maintien de certains paysages et par là de certains écosystèmes. Le tracteur y apparaît alors uniquement comme une représentation de l'activité agricole. 
Figure 3. Dossier documentaire

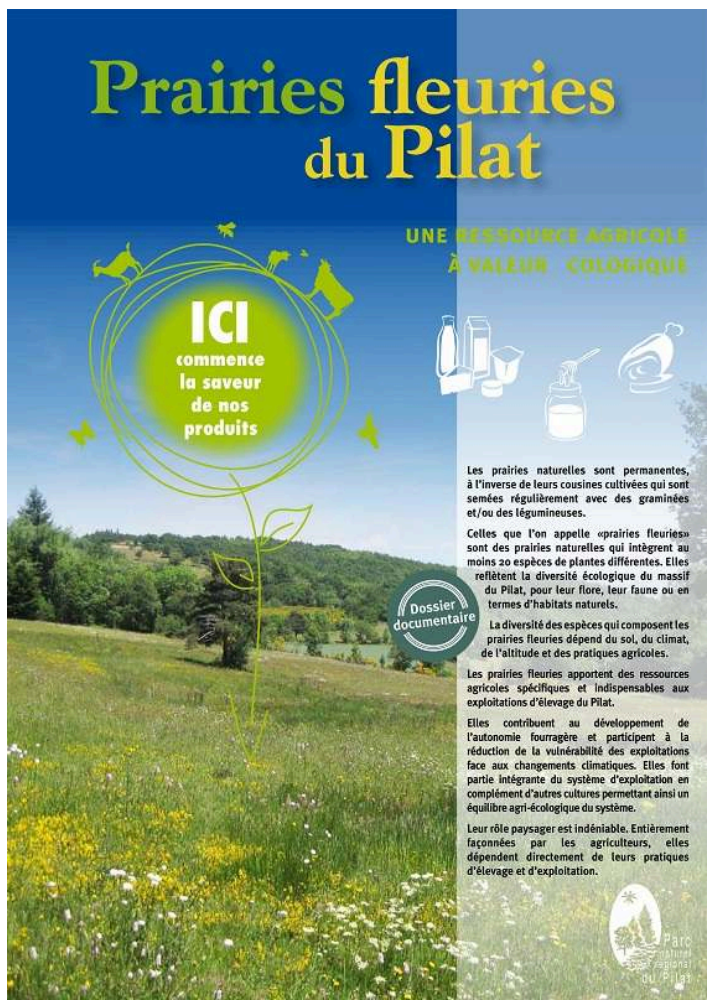

La mise en forme de la couverture de ce Dossier montre une volonté de faire monde commun ${ }^{\mathbf{1 3}}$ : prairies fleuries, abeille et tracteur cohabitent dans l'espace de la page.

Source : image extraite de documents édités par le Parc naturel régional du Pilat.

Autre exemple visuellement emblématique de ce type de reconstruction du tracteur et des pratiques agricoles positives, la page extraite du Dossier documentaire sur l'environnement et l'agriculture montre, de bas en haut, un enchaînement de photographies qui en lui-même représente déjà de la diversité : paysage, zoom sur un lézard, plan moyen sur un muret et un tracteur. Ce dernier est inscrit dans une pratique de fauche raisonnable et le titre de la page "Des bonnes pratiques agricoles " est très explicite. Le PNR y articule considérations écologiques, activités humaines, présence animale, paysages diversifiés et patrimoine vernaculaire avec le muret de pierres sèches. 
Figure 4. Bonnes pratiques, paysages de murets traditionnels et de diversités...

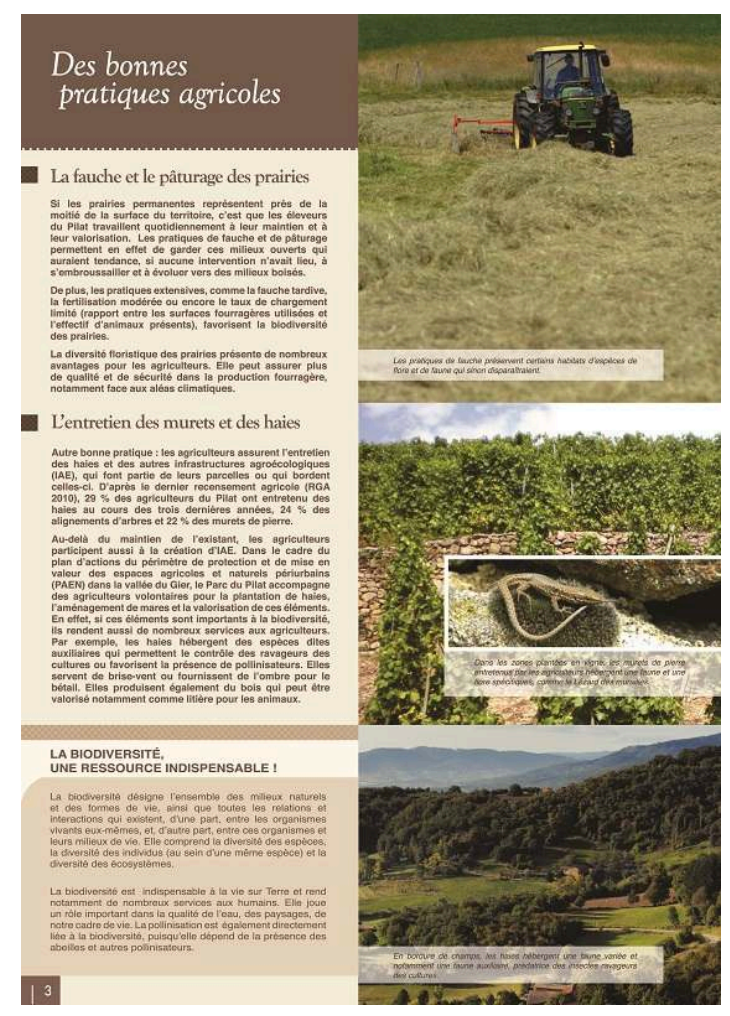

Une mise en page qui joue sur une implicite logique de causalité.

Source : image extraite de documents édités par le Parc naturel régional du Pilat.

36 La maquette de cette page reprend une construction narrative qui met en opposition «bonnes» et «mauvaises» pratiques agricoles. Visuellement l'enchaînement des illustrations peut se comprendre ainsi : de bonnes pratiques agricoles associées à la conservation $\mathrm{du}$ patrimoine vernaculaire permettent la conservation des espèces animales et végétales et aboutissent à des paysages diversifiés et esthétiques. Or, cette conception pourrait être discutée, ne serait-ce qu'en ce qu'elle stigmatise d'autres manières de concevoir l'agriculture et le paysage sur le territoire du Parc tout en figeant celui-ci dans des critères esthétiques immuables (Poinsot, 2008). 
Figure 5. Synthèse des éléments textuels mis en opposition dans les discours à propos des pratiques agricoles

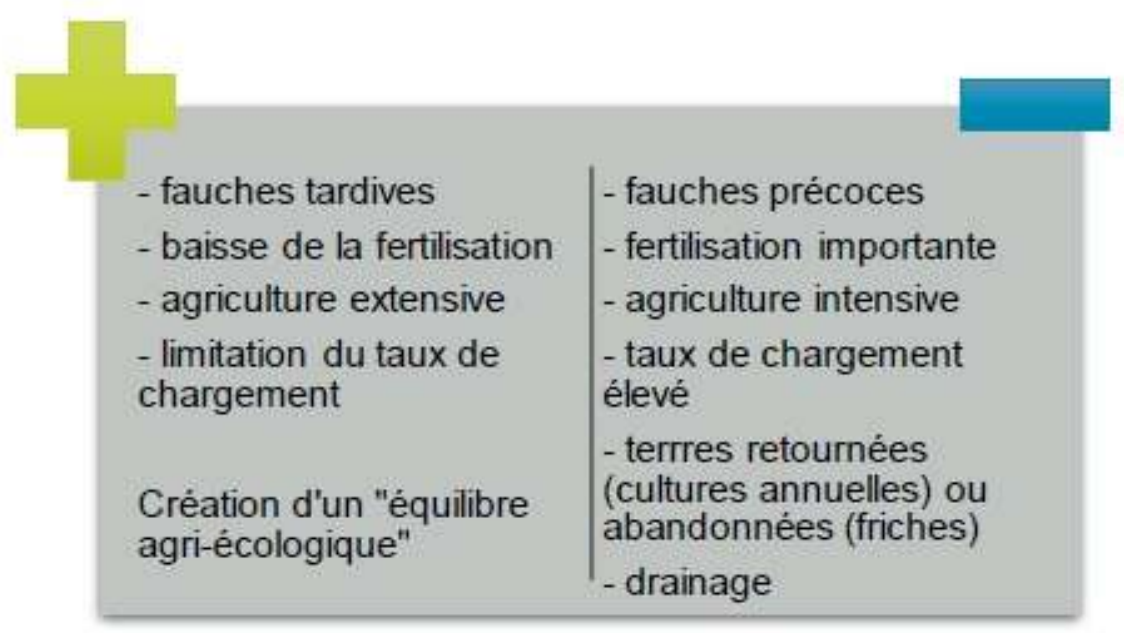

Analyse des Dossiers documentaires du PNR du Pilat.

Source : Émilie Kohlmann

L'utilisation de la figure du tracteur dans la communication du PNR est duelle, comme le paradoxe d'une agriculture en pleine évolution. Dévalorisée et attachée à des paysages peu intéressants esthétiquement ${ }^{14}$, la figure du tracteur peut également être utilisée de façon plus neutre et attaché à des paysages variés. Le Parc a fait le choix de mettre la diversité en avant: diversité des paysages, diversité des pratiques agricoles, diversité des écosystèmes, etc. La thématique de la diversité bénéficie toujours d'une représentation positive. C'est sur ce type de ressort logique qu'est conçue la communication du Parc autour des pratiques et des paysages agricoles. 


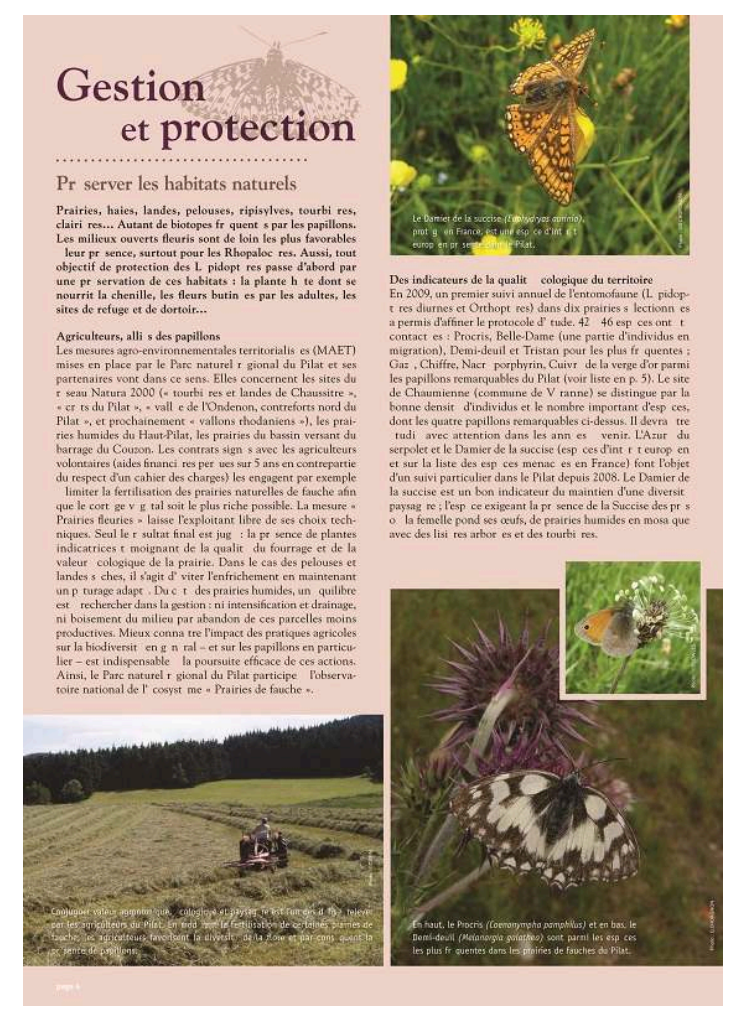

Dossier documentaire sur les papillons du PNR du Pilat.

Source : image extraite de documents édités par le Parc naturel régional du Pilat.

\section{Les prairies fleuries et la double contrainte}

Nous reprendrons dans cette partie le concept de double contrainte emprunté librement à la psychologie de la communication (Watzlawick, P., Beavin, J. H. et Jackson, D.D., 1972). La "double contrainte » ou «double bind» est liée à l'idée de l'injonction paradoxale ${ }^{15}$. Dans le cas des prairies fleuries, on l'élargira à une communication qui veut toucher deux publics a priori incompatibles : les agriculteurs et le grand public. En effet, la "fleur» en elle-même est un objet de communication polysémique, que l'on considère ses aspects esthétiques, les jolies "petites fleurs », ou ses aspects agroécologiques, la « flore ». Pourtant, le PNR du Pilat souhaite les mobiliser ensemble et ne pas abandonner l'un au profit de l'autre. C'est ainsi à travers la figure paysagère des prairies fleuries que l'on peut lire cette double mobilisation.

«Nous, on fait les concours prairies fleuries pour sensibiliser les agriculteurs et valoriser le meilleur équilibre, qui est l'équilibre à la fois écologique et agronomique. [...] ça, ça peut parler éventuellement aux agriculteurs, mais ça ne parle pas du tout aux habitants. Donc quand on parle aux habitants, on parle sur les fleurs qu'on voit dans le paysage, on rentre par l'aspect de ce qui les touche de plus près. » (Chargée de communication du PNR, avril 2011.)

La prairie est, dans le dossier documentaire qui lui est consacré, inscrite comme vu précédemment dans une logique de diversité paysagère et fonctionnelle. L'illustration ci-dessous permet d'appréhender cette diversité. 
Figure 7. Les prairies et la diversité paysagère, fonctionnelle et environnementale

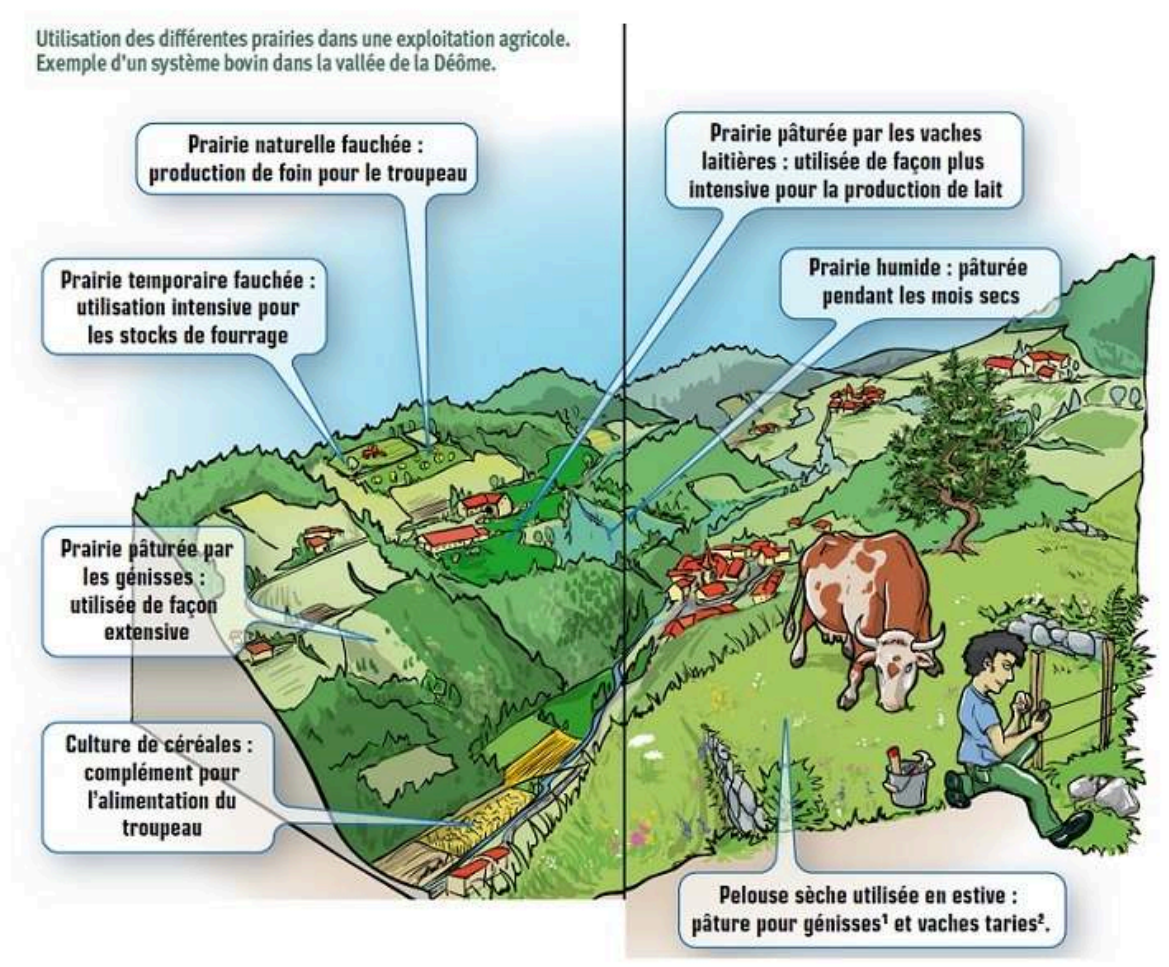

Dossier documentaire sur les prairies fleuries du PNR du Pilat.

Source : image extraite de documents édités par le Parc naturel régional du Pilat. explication fonctionnelle et/ou environnementale: production de foin, pâturage, humide ou sec. Malgré la représentation de l'agriculteur et de la vache en premier plan, le choix graphique s'inscrit dans une dimension paysagère et c'est le paysage qui est reconstruit et décrit à travers sa fonction agricole.

Cependant, loin de s'arrêter là, la rhétorique autour des prairies fleuries se développe à l'extérieur des Dossiers documentaires et notamment à travers une stratégie de communication à destination du grand public et notamment des consommateurs. Le PNR propose ainsi une gamme de supports déclinés à la période estivale, la plus touristique : affiches, sets de table, cartes postales. Ceux-ci, intitulés « Prairies fleuries du Pilat », construisent un discours dans lequel le paysage est utilisé comme espace de transition entre la pratique agricole, l'environnement et la consommation de la production locale.

Dans l'espace gauche de l'affiche (figure 8) se trouvent des représentations iconographiques des «ressources» environnementales et agricoles : chèvre, abeille, vache, veau, papillon, insecte. Ces ressources constituent les pétales d'une fleur qui prend racine dans le paysage. Dans la zone de droite, un pseudo verbatim alterne les appels au «nous » et au « vous » dont on peut supposer qu'il représente les producteurs $\mathrm{du}$ PNR et les consommateurs. Des représentations de "produits" après transformation par l'homme (lait, miel, charcuterie) se situent dans la même zone. La photographie utilisée comme fond d'image, celle du paysage de prairie, devient l'espace où tous ces éléments peuvent se rejoindre. 
Figure 8. La construction de l'affiche du PNR du Pilat sur la thématique des prairies fleuries met en scène le paysage comme espace de transition, de médiation

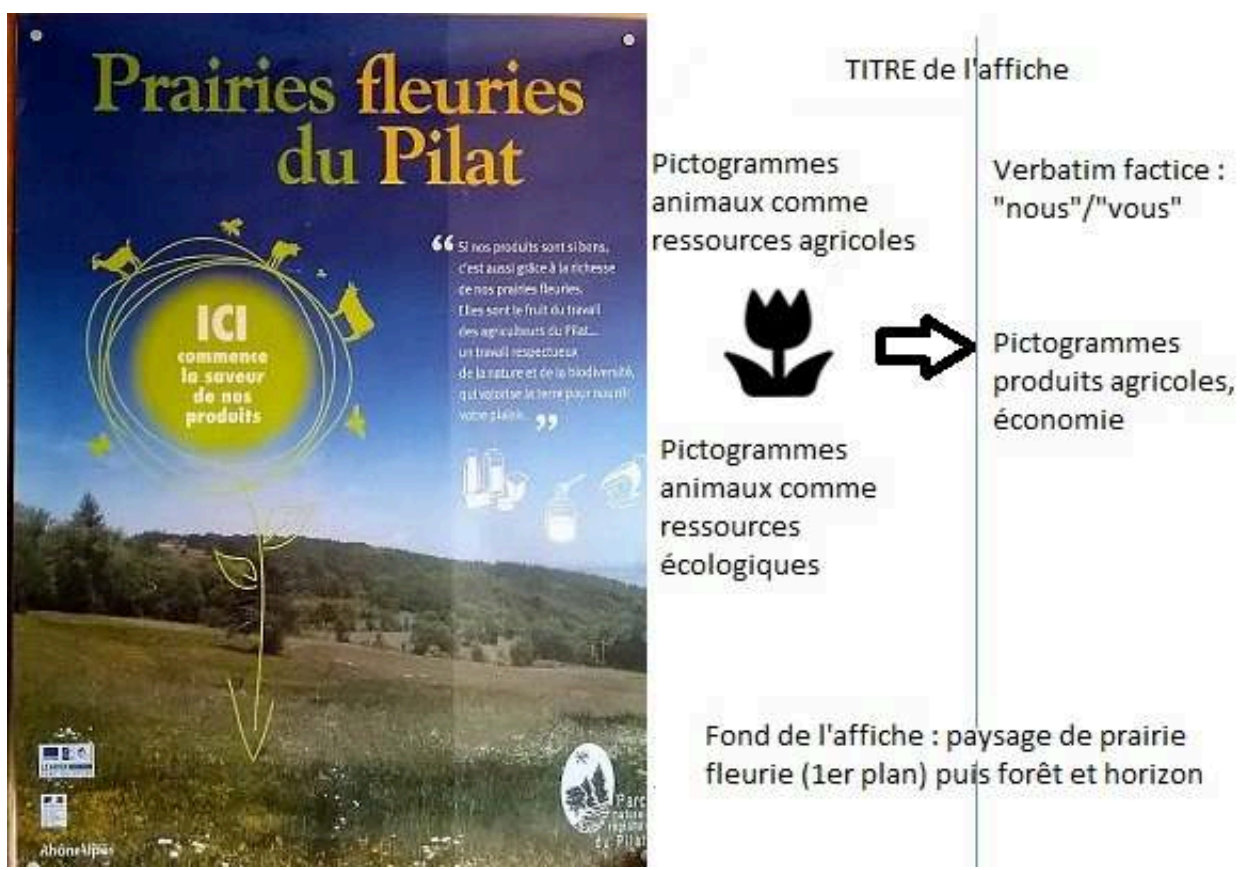

Source : image extraite de documents édités par le Parc naturel régional du Pilat.

Dans le cas de la figure du tracteur, le paysage et le texte qui l'accompagnent le contextualisent et permettent de lui donner un sens négatif ou neutre. Inversement, les prairies fleuries sont l'espace de partage de deux discours a priori antinomiques : celui sur les "petites fleurs" à destination du grand public et celui sur la «flore " à destination des agriculteurs. Le support paysager est utilisé comme transition, zone de médiation entre les deux discours et vise à les faire cohabiter.

Ce constat rejoint la volonté exprimée par la chargée de communication du PNR du Pilat à l'occasion des premières journées de l'Observatoire de la biodiversité du Parc: celle de créer des dispositifs d'échanges pour différents acteurs et discours (Kohlmann, 2016). La thématique des prairies fleuries avait été retenue afin de faire cohabiter des acteurs du territoire qui auparavant ne communiquaient pas : agriculteurs, naturalistes et grand public. Le paysage mis en scène dans ce cas de figure a donc pour objectif de créer un espace de partage entre des publics différents, tout en dressant le portrait de produits issus d'un territoire particulier, dans toute sa diversité. 
Figure 9. Un discours qui vise à convaincre de l'intérêt agricole, environnemental et économique de la diversité paysagère

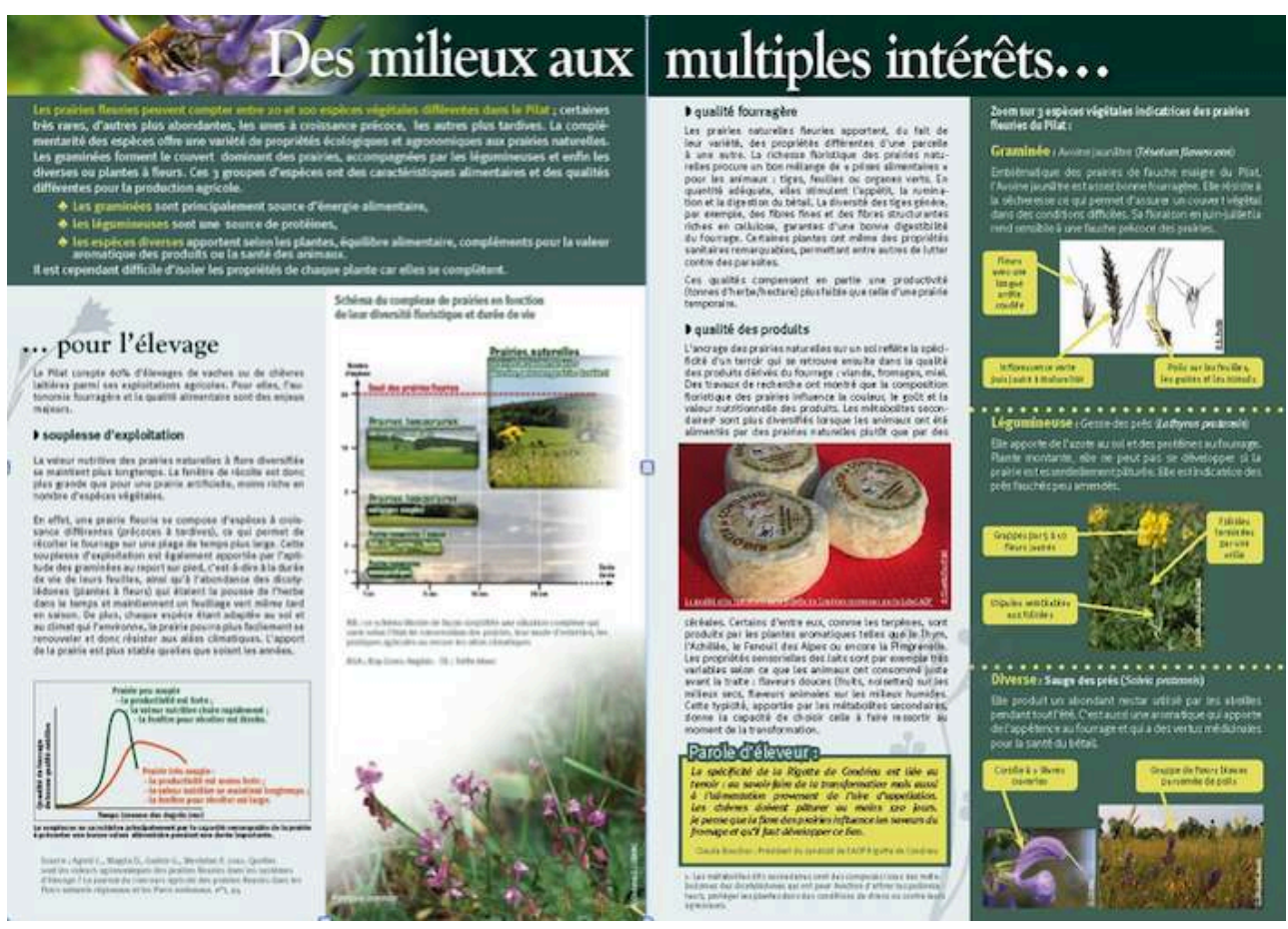

Source : image extraite de documents édités par le Parc naturel régional du Pilat.

\section{Le troupeau et la lande : reconstruction patrimoniale des paysages}

Dernière figure mobilisée dans le paysage agricole qui sera étudiée ici, celle du troupeau. Dans les photographies paysagères, des troupeaux pâturant dans des champs ou des landes sont régulièrement mis en scène. Généralement, la figure de l'agriculteur en est exclue. Comme le tracteur, ces figurations évoquent l'imaginaire agricole (Hamman, 2017) et l'idée d'un espace travaillé par une activité humaine traditionnelle, sans mécanisation apparente. 
Figure 10. Couvertures des deux Dossiers documentaires du PNR du Pilat consacrés à l'agriculture : troupeaux et paysages

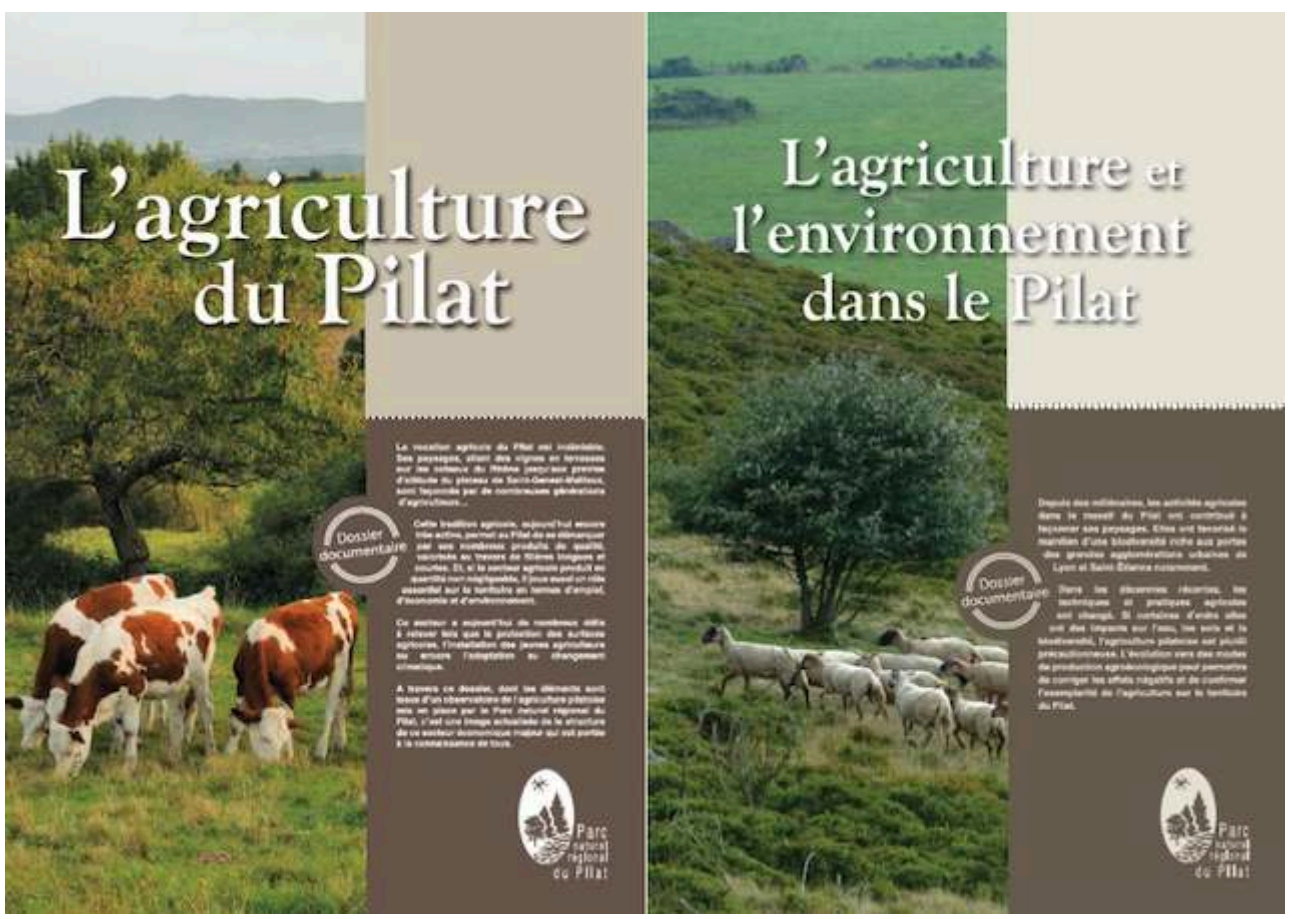

Source : image extraite de documents édités par le Parc naturel régional du Pilat.

Cependant, ce qu'il est intéressant de relever, par comparaison, c'est que, contrairement à celle du tracteur, la figure du troupeau évolue en fonction de l'animal représenté. La vache y apparaît comme un animal familier, inscrite dans une pratique agricole de proximité. Elle est montrée dans des vergers, proche de tracteurs, parfois ramenée à l'étable par son propriétaire.

Chèvres, et surtout moutons, n'ont pas le même traitement. Ils font référence à la volonté de lutte contre l'enfrichement, dans le désir de maintenir des paysages ouverts. Présentés dans des paysages de landes, sur des crêts, dans les fougères ou les ronces, généralement seuls et sans clôture, ils s'inscrivent dans une autre dimension agricole : celle qui associe pratiques agro-environnementales et construction patrimoniale autour de l'estive. Dans un article intitulé « Les moutons au secours de la biodiversité », un agriculteur explique les conditions dans lesquelles l'estive a été relancée dans, et par, le Parc:

« Depuis plus de 20 ans, des troupeaux de moutons montent chaque été pâturer sur le crêt de Chaussitre. "Nous n'avions jusqu'ici pas de tradition d'estive ", remarque J.O., agriculteur aujourd'hui retraité [...]. L'idée est partie d'une boutade à l'occasion d'une réunion en février 1993 [...]. » (Lettre d'information du Parc naturel régional du Pilat : L'Écho des Chirats, septembre 2014, n 13 , spécial 40 ans.)

La réintroduction de la pratique de l'estive est somme toute plutôt récente sur le territoire même à l'échelle de la vie du PNR puisqu'elle existe depuis 20 ans alors que le Parc a fêté ses 40 ans en 2014. Pourtant, dans les deux exemples ci-dessous, elle est mobilisée pour expliquer la diversité paysagère, les pratiques agricoles vertueuses et traditionnelles, la richesse en biodiversité. Elle sert ainsi de support touristique puisque le PNR propose au grand public d'assister à la transhumance des troupeaux et de partager le temps de l'estive avec les agriculteurs ${ }^{16}$. 
Figure 11. Les brebis, l'imaginaire d'estive et la diversité paysagère et écologique affirmée

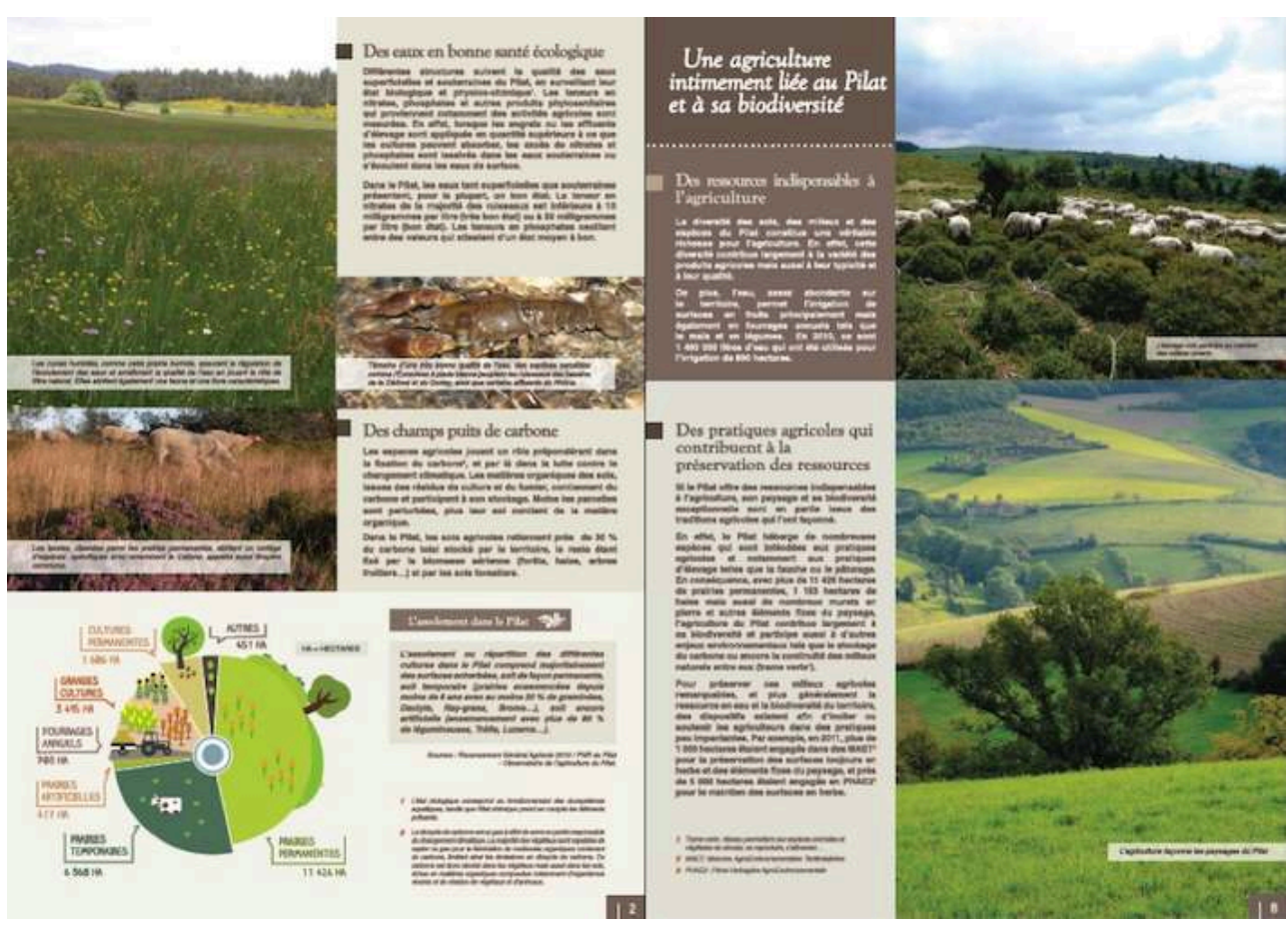

Source : image extraite de documents édités par le Parc naturel régional du Pilat.

49 En définitive, la mise en avant de figures emblématiques d'un certain imaginaire paysan et de ses pratiques traditionnelles (Hamman, 2017), dont l'estive fait partie, relève d'un choix du PNR du Pilat (Davallon, 2006). Rattachées à la thématique de la diversité, et plus précisément, à partir de 2010, de la biodiversité, ces pratiques agricoles traditionnelles sont construites et réutilisées dans la communication comme élément de valorisation. Le PNR y est présenté comme un accompagnateur et un facilitateur de leur développement et de leur maintien.

Cette stratégie de communication semble participer à la construction d'une image de l'action du PNR qui lui permet de se démarquer des autres acteurs (associations, régions, départements, villes portes, etc.) de la protection de l'environnement à l'échelle régionale ${ }^{17}$. Nous avons pu démontrer le fort contexte de concurrence entre les organisations de protection de la nature, tout comme la difficulté pour le Pilat à construire son identité et son image autour d'éléments emblématiques forts (Kohlmann, 2016). Il semble alors que le recours à des éléments construits comme des éléments patrimoniaux ait été mobilisé pour asseoir une communication volontariste et promotionnelle. À la frontière des paysages agricoles de proximité, les landes et les moutons donnent ainsi aux pratiques agricoles une nouvelle dimension. Utiles aux paysages et aux équilibres biologiques, elles deviennent également représentatives de l'histoire d'un territoire particulier.

\section{Conclusion}

51 Les tensions entre les attentes agricoles et environnementales, longtemps considérées comme antinomiques et inconciliables, cherchent à être résolues dans le discours du 
PNR et dans sa mise en scène des paysages agricoles. Ces derniers jouent le rôle d'espace de médiation sur lequel projeter des discours parfois conflictuels.

La « bonne gestion » des paysages et des territoires est construite et utilisée par le Parc afin de se démarquer de ses concurrents, notamment les associations de protection de la nature dans le secteur environnemental. Le rapprochement de la biodiversité et de l'agriculture se fait non pas uniquement dans une perspective économique (la nature fournit des services à l'homme) mais plutôt dans une perspective qui relève d'une construction patrimoniale: c'est parce que le territoire du Pilat a des pratiques agricoles précautionneuses et extensives que sa biodiversité est riche et préservée. L'agriculture est présentée à partir de 2010 dans le Pilat comme un levier pour préserver et maintenir la diversité biologique, mais aussi culturelle.

Se joue également, en creux, le désir de renforcer une identité patrimoniale du territoire. Le Parc ne présente pas un discours où il faut agir pour la biodiversité en réaction à la perte ou à la fragilité de celle-ci : il présente ses actions, et les pratiques des acteurs agricoles tout particulièrement, comme favorables à la diversité.

Plus qu'à un simple greenwashing, procédé de communication qui consiste à construire une nouvelle image écoresponsable d'une entreprise ou d'une société, il s'agit d'une véritable reconstruction de l'image de l'agriculture du Parc. Celle-ci est représentée comme une pratique traditionnelle et relevant de l'identité de son territoire en opposition à une agriculture intensive encore active en périphérie de celui-ci.

\section{BIBLIOGRAPHIE}

Baron, N. et Lajarge, R., Les Parcs naturels régionaux : des territoires en expériences, Versailles, éditions Quæ, 2015.

Berque, A., Médiance : de milieux en paysage, Paris, Belin, 2000.

Bonneuil, C., Fenzi, M., « Des ressources génétiques à la biodiversité cultivée. La carrière d'un problème public mondial ", Revue d'anthropologie des connaissances, vol. 5, n 2, 2011, p. 206-233.

Cevasco, R. et Poggi, G., « L'alpe, l'arbre et le lait : pour une valorisation environnementale et culturelle des produits du terroir de la montagne ligure (Italie) », Sud-Ouest Européen, revue géographique des Pyrénées et du Sud-Ouest, $\mathrm{n}^{\circ}$ 7, Toulouse, Presses universitaires du Mirail, 2000, p. 35-47.

Davallon, J., Le Don du patrimoine : une approche communicationnelle de la patrimonialisation, Paris, Hermès science publications, 2006.

Emprin, C., « Les Dynamiques communicationnelles dans la recherche en écologie : projet et programme de recherche sur la biodiversité ", thèse de doctorat Lyon, École normale supérieure de Lyon, 2012.

Guérin, J.-P., « Préface », Revue de géographie alpine, n 15, 1995, p. 7-9. 
Hamman, P. (dir.), Ruralité, Nature et Environnement : entre savoirs et imaginaires, Toulouse, Éditions Érès, 2017.

Kohler, F., « Diversité culturelle et diversité biologique : une approche critique fondée sur l'exemple brésilien », Natures Sciences et Sociétés, vol. 19, 2011, p. 113-124.

Kohlmann, E., « Communication environnementale et biodiversité dans le Parc naturel régional du Pilat », thèse de doctorat, université de la Réunion, 2016.

Larrère, R., Lizet, B. et Berlan-Darqué, M., Histoire des parcs nationaux : comment prendre soin de la nature, Versailles, éditions Quæ, 2009.

Lepart, J. et Marty, P., « Des réserves de nature aux territoires de la biodiversité : l'exemple de la France », Annales de géographie, t. 115, nº 651, 2006, p. 485-507.

Micoud, A., Laneyrie, P. et Banville, E. de, « Fonctions et Enjeux des parcs naturels régionaux périurbains : l'exemple du Parc du Pilat », Saint Étienne, Cresal, 1977.

Monseigne, A., « Faire monde commun : du discours à l'acte ", Recherches en communication, $\mathrm{n}^{\circ} 42$, 2016, p. 7-17.

Poinsot, Y., Comment l'agriculture fabrique ses paysages : un regard géographique sur l'évolution des campagnes d'Europe, des Andes et d'Afrique noire, Paris, Éditions Karthaka, 2008.

Poux, X. (dir.), Agriculture, Environnement et Territoires : quatre scénarios à l'horizon 2025, Paris, La Documentation française, 2006.

Roué, M., « ONG, peuples autochtones et savoirs locaux : enjeux de pouvoir dans le champ de la biodiversité ", Revue internationale de sciences sociales, vol. 4, n 178, 2003, p. 597-600.

Ruiz, J. et Domon, G. (dir.), Agriculture et Paysage : aménager autrement les territoires ruraux, Montréal, Les Presses de l'université de Montréal, 2014.

Watzlawick, P., Beavin, J. H. et Jackson, D. D., Une logique de la communication, Paris, Éditions du Seuil, 1972.

\section{NOTES}

1. Convention téléchargeable sur le site de la Convention européenne du paysage: https:// www.coe.int/fr/web/conventions/full-list/-/conventions/treaty/176, consulté le 14 décembre 2017.

2. Le concept de «double contrainte » est tiré de la psychologie de la communication et sera développé en troisième partie de cet article.

3. Information disponible sur: https://www.parc-naturel-pilat.fr/le-parc-naturel-regional/unterritoire-labellise/histoire-du-parc-du-pilat/, consulté le 14 décembre 2017.

4. Information disponible sur: https://www.parc-naturel-pilat.fr/un-territoire/paysages/, consulté le 14 décembre 2017.

5. Les chargés de mission en biodiversité, Natura 2000, en agriculture, en tourisme, en communication ont été interrogés, ainsi que leur pendant politique (élu.e.s au bureau du Parc), la présidente et la directrice du PNR en fonction à cette époque. (cf. Kohlmann, 2016).

6. Elle emploie ici le passé pour expliquer que depuis ils ont conduit une stratégie de communication pour essayer d'inverser la tendance et de montrer leur richesse notamment en matière de biodiversité. 
7. Ce document est téléchargeable sur le site du Parc naturel régional du Pilat: https:// www.parc-naturel-pilat.fr/le-parc-naturel-regional/un-projet-la-charte/la-charte-

objectif-2025/, consulté le 14 décembre 2017.

8. Le reste du territoire ligérien est plus propice à une agriculture intensive.

9. L'année 2010 était l'année internationale de la biodiversité (ONU), ce qui a eu de nombreuses conséquences communicationnelles pour les institutions à vocation environnementale (Kohlmann, 2016) et scientifique (Emprin, 2012).

10. 3 numéros de cette collection sont consacrés à la thématique agricole : «L'agriculture dans le Pilat », « Agriculture et environnement » et « Prairies fleuries : des ressources agricoles à valeur écologique ».

11. En 1872, le parc national de Yellowstone est créé par le Congrès des États-Unis, http:// www.parcsnationaux.fr/fr/des-decouvertes/les-parcs-nationaux-de-france/lhistoire-des-parcsnationaux-de-france, consulté le 14 décembre 2017.

12. La loi Beauquier en 1906 et la loi du 2 mai 1930 permettent ainsi le classement et la protection des sites en fonction de critères esthétiques.

13. "Faire monde commun" » est une notion en communication développée notamment dans un dossier de Recherches en communication, qui la définit comme « [la] traduction active d'un faire ensemble » (Monseigne, 2016, p. 10).

14. Julie Ruiz et Gérald Domon (2014, p. 255) définissent les zones d'intensification agricole comme sans attraits visuels marqués et sans « consensus social quant à leur valeur ».

15. L'exemple proposé est souvent le suivant : « sois spontané ! » puisque l'ordre donné contredit l'injonction faite.

16. Cette activité fait partie des «Rendez-vous du Parc du Pilat », ensemble d'animations qui se déroulent entre mai et octobre.

17. Sur l'enjeu de la figuration pour les PNR, voir Baron et Lajarge (2015), partie III et particulièrement le chapitre 9 (p.177-197).

\section{RÉSUMÉS}

Cet article s'inscrit en sciences de l'information et de la communication et s'appuie sur l'analyse des documents de communication produits par le Parc naturel régional du Pilat de 2010 à 2015. Celle-ci est centrée sur le recours aux paysages agricoles pour illustrer certains discours et actions du Parc. Leur représentation dans des documents de communication institutionnelle met en avant le rôle de médiateur de cette collectivité. En effet, les représentations paysagères agricoles mobilisées servent d'espace de médiation sur lequel s'inscrivent des discours régulièrement et historiquement conflictuels : défense de l'environnement, conservation du patrimoine, développement économique et pratiques professionnelles des agriculteurs. Ainsi, la stratégie de communication du Parc permet, en passant par le paysage agricole et la nature ordinaire, de réconcilier différents acteurs de son territoire : habitants, touristes, naturalistes, agriculteurs, etc.

This article is a part of an information and communication science research study and is based on the analysis of communication documents produced by the Pilat Regional Nature Park from 2010 to 2015. The study focuses on how landscapes are used to illustrate certain principles and actions on the part of the park authorities. Their representation in institutional communication 
documents highlights the mediating role played by these authorities. The representations of farming landscapes used serve in mediating between positions which are frequently and historically in conflict, such as the defense of the environment, heritage conservation, economic development, and professional farming practices. The communication strategy of the park authorities makes it possible, by employing representations of the farming landscape and of ordinary nature, to reconcile the positions of the park's different stakeholders such as local inhabitants, tourists, nature lovers and farmers.

\section{INDEX}

Mots-clés : communication, parc naturel régional, biodiversité, pratiques agricoles, patrimoine paysager

Keywords : communication, regional nature park, biodiversity, farming practices, landscape heritage

\section{AUTEUR}

\section{ÉMILIE KOHLMANN}

Émilie Kohlmann est docteure en sciences de l'information et de la communication (laboratoire LCF, université de La Réunion). Ses domaines de recherche sont la communication environnementale, la biodiversité, la médiation et les dispositifs participatifs. Depuis le $1^{\mathrm{er}}$ septembre 2017, elle est Ater à l'IUT 2 de Grenoble (laboratoire Gresec).

emilie.kohlmann[at]laposte[dot]net 